\title{
Research on the Economic Spatial Connection in Coastal and Hinterland of Shandong Province
}

\author{
Yuanyuan Li \\ Tianjin University of Finance and Economics \\ Tianjin, China
}

\begin{abstract}
This paper takes 10 inland cities and 7 coastal cities in Shandong Province as the research object, and uses the Gravity Model to explore the spatial-temporal evolution of economic linkage strength and economic connection intensity between coastal and hinterland of Shandong Province from the perspective of population and gross value of production. Through empirical analysis, it is concluded that the economic spatial connection strength of the coastal and hinterland of Shandong Province is becoming stronger and stronger as a whole, each coastal (inland) city is different from the inland (coastal) cities in economic spatial connection and at the same time, and the economic spatial intensity of the coastal and hinterland of Shandong Province is generally not very high. The reasons are briefly analyzed. Based on this, some suggestions were made.
\end{abstract}

Keywords-coastal and hinterland; economic spatial connection; Gravity Model; Shandong Province

\section{INTRODUCTION}

The increasingly active regional economic linkages can promote the division of labor and cooperation in the region and are conducive to the coordinated development of the region. Therefore, strengthening regional spatial links has become an important scientific choice for the sustainable development of all economic regions. In a long period of time, China's development of the ocean and terrestrial economy is independent of each other. People lack the correct understanding and in-depth study of the strategy for the overall development of the sea and the land, and thus the characteristics of "land and land dualization" emerge. The "Twelfth Five-Year Plan" outlines that "To advance marine development, we must adhere to the overall planning of the sea and land, and regard the coordinated development of land and sea as an important part of the transformation and upgrading and improvement of the core competitiveness of coastal areas". The report of the party's Nineteenth Congress also pointed out that "We must adhere to the overall planning of the sea and the land and accelerate the building of a marine power." With the implementation of the strategy of building a strong ocean nation and overall planning of land and sea, the relationship between land and sea systems has continued to deepen. It is of great significance to explore the economic spatial connection strength between coastal and hinterland of Shandong Province, and to promote the sustainable economic and social development of Shandong
Province, realize the overall sea-land cooperation, and enhance the economic strength of Shandong Province.

Current studies on the relationship between coastal and hinterland systems or land-sea integration: The first is the theoretical discussion of the basic concept connotation, for example, the integration of land and sea resources through the optimization of resource allocation to coordinate the relationship between land and sea resources and industrial economics is an important principle of the ocean development strategy, and it is a development strategy and model for the realization of land and sea overall planning. The other is the regional comparison and empirical test of the ocean and terrestrial systems, for example, using regional economics, economic geography, and systemic synergy theories, three major hotspots are focused on ecological environmental protection and pollution control, industrial resource development, and factor allocation, as well as the level and structure of economic development. The status quo and major differences of land and sea systems are analyzed. And the existing problems, the use of sea-land integration theory proposed regional coordinated development strategies and recommendations.

At present, domestic and foreign scholars mainly focus on the spatial linkage of urban agglomeration. Hidenobu Matsumoto studied the strength of aviation flow based on the Gravity Model under distance, population, and economic indicators, and then analyzed the network characteristics of the urban agglomeration. Guo-qiang Shen focuses on the gravitational attraction of city nodes. The domestic research on regional spatial connections focuses on interregional linkages. The focus of research is on the spatial relationship between the city and its region or a larger area, and it focuses on the study of spatial transport linkages. In terms of research methods, the Gravity Model and the urban flow intensity model are mainly used, but the Gravity Model is less applied.

Based on the perspective of regional spatial economic relations, this paper uses the Gravity Model to investigate the spatial-temporal evolution of economic linkage strength and economic linkage strength between Shandong coastal provinces and hinterland with 10 inland cities and 7 coastal cities in Shandong Province to achieve the coordinated development of the coastal and hinterland economy of Shandong Province to provide theoretical guidance and decision-making reference. 


\section{STUdy AREA AND METHOD}

\section{A. The Study Area}

Shandong Province is located on the eastern coast of China and on the lower Yellow River. The territory includes the peninsula and the interior. There are 17 prefecture-level cities in Shandong Province, including 7 coastal cities and 10 inland cities. Qingdao, Yantai, Weihai, Weifang, Rizhao, Dongying and Binzhou are all coastal city. Jinan, Zibo, Zaozhuang, Jining, Tai'an, Laiwu, Linyi, Dezhou, Liaocheng and Heze are all inland city. Shandong Province is one of the most economically developed provinces in China, one of the provinces with the strongest economic strength in China, and one of the fastest growing provinces in China.

\section{B. Research Method}

The Gravity Model originates from Newton's Gravity Model and is a very important physical model. Later, the Gravity Model was applied to the field of economic research and gradually improved, forming the basic Gravity Model often used in regional science and location theory. ${ }^{123}$ The basic Gravity Model is

$$
I_{i j}=k \frac{M_{i} M_{j}}{G_{i j}^{b}}
$$

Among them, $I_{i j}$ represents the economic spatial connection strength between city $\mathrm{i}$ and city $\mathrm{j}, M_{i}$ and $M_{j}$

${ }^{M}{ }_{j}$ represent the population size or GDP of city i and city $\mathrm{j}$, respectively, ${ }^{{ }^{i j}}$ is the distance between city $\mathrm{i}$ and city $\mathrm{j}$; $k$ is the empirical constant and $b$ is the distance coefficient. The Gravity Model can not only express the spatial relationships between regions more intuitively, but can further quantify them and measure the strength of the connections between regions.

Comprehensive study of the use of Gravity Model by various scholars, some scholars from the population scale of the two places, some scholars from the GDP of the two places to study the strength of economic spatial connection, and some scholars to improve the Gravity Model from The Industrial Development Index sets out to study the strength of economic spatial linkages between the two cities. This article is based on a regional perspective, combining population and gross value from the perspective of population and gross value of production, and using Gravity Models to study the economic spatial linkage between coastal and hinterland of Shandong Province. The model

$$
\text { used in this paper is: } I_{i j}=k \frac{\sqrt{P_{i} G_{i}} \times \sqrt{P_{j} G_{j}}}{D_{i j}^{b}}
$$

$I_{i j}$ refers to the strength of economic connections between coastal (inland) city $\mathrm{i}$ and inland (coastal) city $\mathrm{j}$, $P_{i}$ and $P_{j}$ are the urban population in the cities of city $i$ and city $\mathrm{j}$ (ten thousand people); $G_{i}$ and $G_{j}$ are the sum of the total production value of the secondary and tertiary industries of city $\mathrm{i}$ and $\mathrm{j}$ (100 million yuan), $D_{i j}$ is seen as the distance between the coastal (inland) city $\mathrm{i}$ and the inland (coastal) city $\mathrm{j}$; Summarize the experience of previous scholars, and to facilitate the calculation, the empirical constant $k$ is taken as 1 and the distance coefficient $b$ is taken as 2 .

In data selection, most of the people engaged in agricultural production are rural people because most of the close economic space connections between the two places do not include primary industries such as agriculture. Therefore, in this paper, we use the urban population and the total GDP of the second industry and the third industry to calculate the economic strength between cities. At present, because of its economical, convenient, and rapid performance, automobiles have become the major transportation carriers for regional resource flows. Moreover, Shandong has a complete transportation network and convenient transportation. Therefore, this paper selects the freeway distance to measure the distance between the two places. The data comes from Shandong Statistical Yearbook.

\section{EMPIRICAL RESULTS AND ANALYSIS}

This paper chooses to combine the population with the GDP from the perspective of the region and from the perspective of population and gross production. Using the gravity model, we calculate the economic spatial intensities of coastal areas and hinterland of Shandong Province in 2011 and 2016 respectively, to explore the evolution of the economic spatial connection intensity between the coastal and hinterland of Shandong Province in time and space.

\footnotetext{
Tobias G, Franz R and Armin H, Gravity models for airline passenger volume estimation, Journal of Air Transport Management,2007,pp.175-183.

2 Li Hongjin, Li Shenghui, Gravity Model Based on Spatial Connection of Urban Agglomeration Economies: An Empirical Study of the Pearl River Delta City Group, Journal of South China University of Technology(Social Science Edition), 2011.

3 Jiang Tianying, Xie Min and Liu Gang, Spatial Linkage of Regional Innovation Output Based on Gravity Model: A Case Study in Zhejiang Province, Scientia Geographica Sinica,2014.
} 
A. Temporal Characteristics of Economic Spatial Linkage Strength Between Coastal and Hinterland

TABLE I. ECONOMic SPace Strength in COASTAL AND Hinterland of Shandong Province IN 2011

\begin{tabular}{|c|l|l|l|l|l|l|l|l|l|l|}
\hline & Jinan & Zibo & Zaozhuang & Jining & Tai'an & Laiwu & Linyi & Dezhou & Liaocheng & Heze \\
\hline Qingdao & 19.04 & 19.64 & 4.49 & 8.14 & 9.95 & 4.11 & 21.06 & 4.58 & 4.58 \\
\hline Yantai & 7.95 & 7.53 & 1.49 & 2.73 & 2.83 & 1.03 & 5.30 & 2.20 & 2.21 \\
\hline Weihai & 2.56 & 2.26 & 0.51 & 0.93 & 0.94 & 0.33 & 1.72 & 0.74 & 0.74 \\
\hline Weifang & 37.93 & 77.58 & 3.71 & 6.25 & 8.84 & 4.31 & 19.04 & 7.17 & 7.05 & 0.43 \\
\hline Rizhao & 4.16 & 2.96 & 1.91 & 3.40 & 2.79 & 1.01 & 15.06 & 0.96 & 1.00 & 3.02 \\
\hline Dongying & 12.24 & 22.05 & 1.09 & 2.17 & 3.00 & 1.08 & 4.94 & 4.04 & 2.47 \\
\hline Binzhou & 23.93 & 60.65 & 1.37 & 3.38 & 5.42 & 2.42 & 4.54 & 6.70 & 4.68 & 1.06 \\
\hline
\end{tabular}

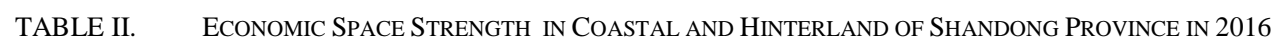

\begin{tabular}{|c|l|l|l|l|l|l|l|l|l|l|}
\hline & Jinan & Zibo & Zaozhuang & Jining & Tai'an & Laiwu & Linyi & Dezhou & Liaocheng & Heze \\
\hline Qingdao & 36.14 & 42.15 & 9.59 & 14.40 & 22.70 & 7.61 & 46.66 & 10.93 & 10.01 & 8.65 \\
\hline Yantai & 14.50 & 15.52 & 3.07 & 4.64 & 6.21 & 1.82 & 11.29 & 5.04 & 4.64 \\
\hline Weihai & 4.95 & 4.95 & 1.11 & 1.68 & 2.19 & 0.62 & 3.88 & 1.79 & 1.66 \\
\hline Weifang & 68.36 & 158.0 & 7.53 & 10.49 & 19.14 & 7.57 & 40.05 & 16.25 & 14.63 & 7.66 \\
\hline Rizhao & 8.48 & 6.82 & 4.37 & 6.47 & 6.85 & 2.00 & 35.85 & 2.47 & 2.34 & 3.23 \\
\hline Dongying & 24.34 & 49.54 & 2.43 & 4.02 & 7.18 & 2.09 & 11.45 & 10.10 & 5.65 & 2.97 \\
\hline Binzhou & 46.23 & 132.4 & 2.99 & 6.09 & 12.59 & 4.55 & 10.24 & 16.26 & 10.41 & 4.26 \\
\hline
\end{tabular}

From the data in "Table I" and "Table II", it can be seen that the economic spatial connection strength between the coastal and hinterland of Shandong Province is getting stronger and stronger. The strength of the contact in 2016 is basically two to three times the strength of the contact in 2011. Weifang-Zibo's spatial economic linkage strength reached 158.0 in 2016. According to the empirical data, the size of the economic spatial relationship between coastal and inland cities is classified. The economic spatial connection strength is above 100 for the first level, between 10 and 100 for the second level, and below 10 for the third level. According to this level, in 2011, the economic ties between the coastal areas and hinterland of Shandong Province mostly belonged to the third level, as many as 60 , and the first level did not. In 2016, there were two first-level city combinations. Weifang-Zibo's spatial economic linkage strength reached 158.0, Binzhou-Zibo's space economic contact intensity reached 132.4. The number of the third level has been greatly reduced. Compared with 2011, it has decreased by nearly $1 / 3$, and the number of the second level has increased. In short, the strength of economic space in the coastal and hinterland of Shandong Province has become stronger.

\section{B. Characteristics of The Spatial Intensity of Economic Space Between Coastal and Inland Areas}

TABLE III. RANKING OF ECONOMIC INTENSITY OF COASTAL AND INLAND AREAS IN 2016

\begin{tabular}{|l|llllllll|}
\hline \multicolumn{1}{|c|}{ City } & \multicolumn{7}{|c|}{ Economic strength weakens from left to right } \\
\hline Qingdao & Linyi Zibo Jinan & Tai'an Jining Dezhou Liaocheng & Zaozhuang & Heze & Laiwu \\
\hline Yantai & Zibo Jinan Linyi & Tai'an Dezhou Jining & Liaocheng & Heze & Zaozhaung & Laiwu \\
\hline Weihai & Jinan Zibo Linyi & Tai'an Dezhou Jining & Liaocheng & Heze & Zaozhaung & Laiwu \\
\hline Weifang & Zibo Jinan Linyi Tai'an Dezhou Liaocheng Jining Heze & Laiwu Zaozhuang \\
\hline Rizhao & Linyi Jinan Tai'an Zibo Jining Zaozhaung Heze Dezhou Liaocheng Laiwu \\
\hline Dongying & Zibo Jinan Linyi Dezhou Tai'an Liaocheng Jining Heze Zaozhaung Laiwu \\
\hline Binzhou & Zibo Jinan Dezhou Tai'an Linyi Liaocheng Jining Laiwu Heze Zaozhaung \\
\hline
\end{tabular}

As can be seen from "Table III", from the point of view of the coastal area, Laiwu, Zaozhuang, and Heze are in a weak state of economic strength, Linyi, Zibo and Jinan have a high degree of economic spatial connection with coastal cities in contact. From Table II, it can be seen that the Strength difference of economic linkages between Qingdao and inland areas is not far behind and at a relatively high level, the Strength difference of economic linkages between Yantai, Weihai and inland areas is not big but at a relatively low level. The overall strength of economic ties between Weifang, Binzhou and inland areas is relatively high but the strength difference of economic linkages is very high. Weifang-Zibo has the strongest Economic linkage strength, reaching 158.0, while the Weifang-Zaozhuang economic linkage strength is only 7.53.There is a difference of 150.4 . Binzhou-Zibo economic linkage strength reached 132.4, while Binzhou-Zaozhuang economic linkage strength was only 2.99 , there is a difference of 129.41.The Strength difference of economic linkages between Rizhao, Dongying and inland areas is in the middle and at a relatively centered level. 
TABLE IV. RANKING OF ECONOMIC INTENSITY OF INLAND AND COASTAL AREAS IN 2016

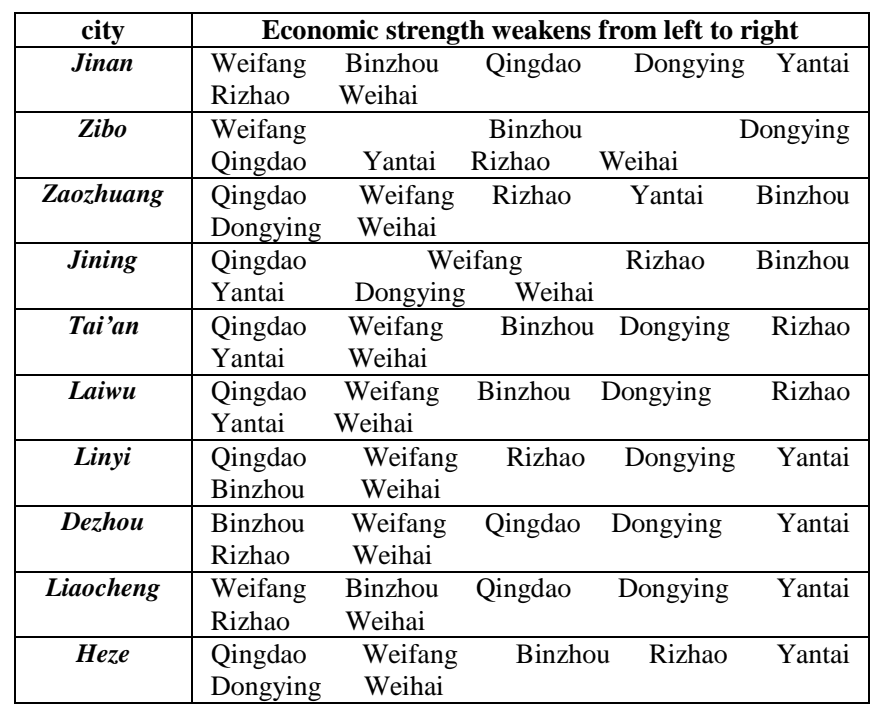

From "Table IV", it can be seen that from the perspective of the interior, Weihai has always been in the weakest state of economic ties with the economic space in the coastal regions. The economic ties between Rizhao, Yantai, and inland areas are also relatively weak. Weifang and Qingdao have the strongest degree of economic ties with inland areas. The difference in the economic linkage between JinanWeifang (68.36) and Jinan-Weihai (4.95) is 63.41; the difference in the economic linkage between Zibo-Weifang (158.0) and Zibo-Weihai (4.95) is 153.05; Linyi-Qingdao (46.66) and Linyi-Weihai (3.88) .The difference in the strength of economic ties is 42,78 . The economic spatial intensities of Taian, Liaocheng, and coastal regions are not far apart and are relatively centered. The intensity of economic linkages between other inland cities and coastal cities is not wide and the strength of economic ties is generally weak.

In addition to this, the intensity of economic spatial connection between the coastal areas and the hinterland of Shandong Province is generally not high, from a whole point of view, and most of the economic linkage strengths are concentrated below 10, there are 7 more combinations in cities below 2 and the Laiwu-Weihai economic linkage strength is only 0.62.Therefore, it is also a long-term task for Shandong Province to strengthen spatial and maritime economic links and achieve sea and land coordination.

TABLE V. Distance Between Coastal Cities And Inland Cities in Shandong Province(KM)

\begin{tabular}{|c|c|c|c|c|c|c|c|c|c|c|}
\hline & Jinan & Zibo & Zaozhuang & Jining & Tai'an & Laiwu & Linyi & Dezhou & Liaocheng & Heze \\
\hline Qingdao & 351 & 265 & 415 & 421 & 334 & 278 & 281 & 457 & 465 & 530 \\
\hline Yantai & 453 & 357 & 600 & 606 & 522 & 464 & 467 & 550 & 558 & 715 \\
\hline Weihai & 514 & 419 & 662 & 668 & 583 & 526 & 528 & 611 & 619 & 777 \\
\hline Weifang & 207 & 111 & 380 & 400 & 295 & 226 & 246 & 304 & 312 & 457 \\
\hline Rizhao & 330 & 300 & 280 & 286 & 277 & 247 & 146 & 438 & 438 & 395 \\
\hline Dongying & 224 & 128 & 432 & 417 & 311 & 278 & 297 & 249 & 324 & 474 \\
\hline Binzhou & 164 & 79 & 393 & 342 & 237 & 190 & 317 & 198 & 241 & 399 \\
\hline
\end{tabular}

As can be seen from "Table V", the distance between Weihai and the inland cities is the furthest away from other coastal cities. This can explain to a certain extent, "From the perspective of the interior, Weihai has always been in the weakest state of economic ties with the economic space in the coastal regions." Therefore, it can also explain why there is weak economic spatial links between Rizhao, Yantai, and the inland cities .In the economic and spatial connection between the inland cities and each coastal city, Heze, Laiwu and Zaozhuang are in a weak state of connection, which is also related to their distance from the coastal cities.In addition, the distance between Weihai and Linyi is almost the same as the distance between Weihai and Laiwu, but in 2016, Weihai-Linyi (3.88) and Weihai-Laiwu (0.62) have a large gap in economic strength. This is related to the economic level of various places. Coastal (inland) cities with high economic levels have a strong ability to promote radiation, and thus have strong economic spatial links with inland (coastal) cities.

\section{CONCLUSION}

This paper takes the 10 inland cities and 7 coastal cities in Shandong Province as the research units, and uses the gravity model to explore the temporal and spatial evolution of the economic linkage strength and economic connection strength between the coastal and hinterland of Shandong Province. Through empirical analysis, it is concluded that the strength of economic spatial connection between the coastal and hinterland of Shandong Province is becoming stronger and stronger; at the same time, the spatial intensity of economic space between the coastal and hinterland of Shandong Province is generally not high, and the gap is relatively large. Hereby, the following suggestions are proposed: Adhere to the principle of "land and land coordination", strive to reduce or even eliminate the barriers to development of various industries in the coastal and inland areas, establish distinctive industrial chains, strengthen the economic strength of cities, and increase the gravitational basis of economic ties; Continue to improve the transportation network system, build large-scale transportation in Shandong Province, and strive to eliminate the hindrance of geographic distance factors to economic spatial connections, reduce the time necessary for resource circulation, and provide the basis for economic interaction between the coastal and inland areas; Establishing an overall development mechanism for the purpose of joint development of regional land- and sea-systems. Under the premise of ensuring the development of the respective advantageous industries in the coastal (inland) regions, we will strengthen the tilt of each industry's underdeveloped 
inland (coastal) cities and strive to form coastal and the new pattern of mutual promotion, close contact and coordinated development in the hinterland.

\section{REFERENCES}

[1] Tobias G, Franz R and Armin H, Gravity models for airline passenger volume estimation, Journal of Air Transport Management,2007, pp.175-183.

[2] Li Hongjin, Li Shenghui,Gravity Model Based on Spatial Connection of Urban Agglomeration Economies: An Empirical Study of the Pearl River Delta City Group,Journal of South China University of Technology(Social Science Edition), 2011.

[3] Jiang Tianying, Xie Min and Liu Gang,Spatial Linkage of Regional Innovation Output Based on Gravity Model: A Case Study in Zhejiang Province, Scientia Geographica Sinica, 2014.

[4] CAO Ke, Discussion on the Connotation and Target of Sea-Land Coordination, Territory \& Natural Resources Study, 2012.

[5] Liu Jianzhao and Gao Suying, The Research of Beijing-Tianjin-Hebei Urban Agglomerations' Spatial Connection Based on Urban Relation Intensity and Urban Flow,Areal Research and Development, 2013.

[6] Zhou Ting, Qiu Fangdao, Zhu Chuangeng, Zhuang Jing, Cai Ai-jun, Sun Dongqi and Fang Xue,Industrial Spatial Association Characteristics in Huaihai Economic Zone, Scientia Geographica Sinica, 2010. 